\title{
Anticipatory Signatures of Voluntary Memory Suppression
}

\author{
Simon Hanslmayr, Philipp Leipold, Bernhard Pastötter, and Karl-Heinz Bäuml \\ Department of Experimental Psychology, Regensburg University, 93053 Regensburg, Germany
}

Voluntary memory suppression can keep unwanted memories from entering consciousness, inducing later forgetting of the information. In the present study, we searched for the existence of anticipatory processes, mediating such voluntary memory suppression. Using the think/no-think paradigm, subjects received a cue whether to prepare to think of a previously studied cue-target pair or whether to not let a previously studied cue-target pair enter consciousness. Examining event-related potentials, we identified two electrophysiological processes of voluntary memory suppression: (1) an early anticipatory process operating before the memory cue for a to-be-suppressed memory was provided, and (2) a later process operating after memory cue presentation. Both ERP effects were due to a decreased right frontal and left parietal positivity. They were positively related and predicted later forgetting. The results point to the existence of anticipatory processes, mediating voluntary memory suppression.

\section{Introduction}

Control processes can be voluntarily engaged to keep unwanted memories from entering consciousness. In everyday life, such voluntary memory suppression is needed to keep our minds free from emotionally straining events (e.g., traumatic experiences) or from neutral outdated information (e.g., the expired computer password). Anticipatory engagement of suppression processes could be highly effective in preparing the system to not let an unwanted memory enter consciousness. Here we present evidence that such processes mediate voluntary memory suppression.

In the laboratory, voluntary memory suppression can be studied using the think/no-think (T/NT) paradigm (Fig. 1a). In this paradigm, subjects study several cue-target pairs and then engage in a T/NT task (Anderson and Green, 2001; Depue et al., 2006). In each trial of the task, the cue item of a cue-target pair is presented, and subjects are instructed to either remember (T condition) or actively suppress any thought (NT condition) about the target. Suppressing a target memory several times during NT trials often leads to impaired subsequent recall of target information, indicating that voluntary suppression of an unwanted memory leads to later forgetting. Imaging studies revealed that voluntary suppression elicits increased activity in prefrontal areas and decreased activity in brain regions processing components of memory representations (Anderson et al., 2004; Depue et al., 2007). This finding suggests that the lateral prefrontal cortex inhibits the neural machinery underlying memory processing, mediating later forgetting of the suppressed information.

Investigating event-related potentials (ERPs) and hemodynamic changes in the hippocampus, previous studies point to the relevance of anticipatory processes during memory formation

\footnotetext{
Received 0ct. 1, 2008; revised Jan. 13, 2009; accepted Jan. 16, 2009.

We thank M. Anderson, M. Wimber, T. Staudigl, and two anonymous reviewers for helpful comments on a previous version of this manuscript.

Correspondence should be addressed to Karl-Heinz Bäuml, Department of Experimental Psychology, Regensburg University, Universitätsstrasse 31，93053 Regensburg, Germany. E-mail: karl-heinz.baeuml@ psychologie.uni-regensburg.de.

DOI:10.1523/JNEUROSCI.4703-08.2009

Copyright $\odot 2009$ Society for Neuroscience $\quad$ 0270-6474/09/292742-06\$15.00/0
}

(Adcock et al., 2006; Otten et al., 2006). In particular, enhanced right frontal positivity seems to index the initiation of, or preparation for episodic retrieval (Düzel et al., 1999; Herron and Wilding, 2006). To date it is unclear whether such anticipatory processes are also triggered when the brain prepares for episodic inhibition, which would point to the initiation of an episodic inhibition mode. To investigate these effects, we adjusted the experimental phase of the T/NT paradigm by presenting the T/NT instruction before the memory cue, allowing subjects to prepare for the upcoming memory cue (Fig. 1a). Concerning the effects on memory cue presentation, our expectations were guided by previous work showing that stronger ERP positivity over frontal and left parietal electrode sites indexes retrieval success and recollection (Allan et al., 1996; Rugg et al., 1998). Such positivity might be downregulated during episodic memory inhibition (Bergström et al., 2007).

Forgetting in the T/NT paradigm is typically not present after a small number of T/NT trial repetitions (Anderson and Green, 2001; Depue et al., 2006), suggesting that the inhibitory processes creating later forgetting are stronger in the late compared with the early T/NT trials. As shown by recent imaging work, neural activation that predicts subsequent forgetting emerges in the later trials of the T/NT phase (Depue et al., 2007). We therefore focused the analysis on the electrophysiological differences between the first and the second half of the T/NT trial repetitions.

\section{Materials and Methods \\ Participants \\ Twenty-four healthy volunteers (mean age: 21.54, range: 19-25; 10 males) participated in the experiment. All participants were right- handed native German speakers with normal or corrected to normal vision. No participant reported any history of neurological disease. All subjects gave their written informed consent and received credit points or monetary reward for participation.}

\section{Materials}

For the cue-target associations, 54 faces with a neutral expression were used as cues, and 54 semantically unrelated words served as targets. The faces were drawn from the AR Face Database by Martinez and Benavente (1998). The word material was drawn from a semantically categorized 

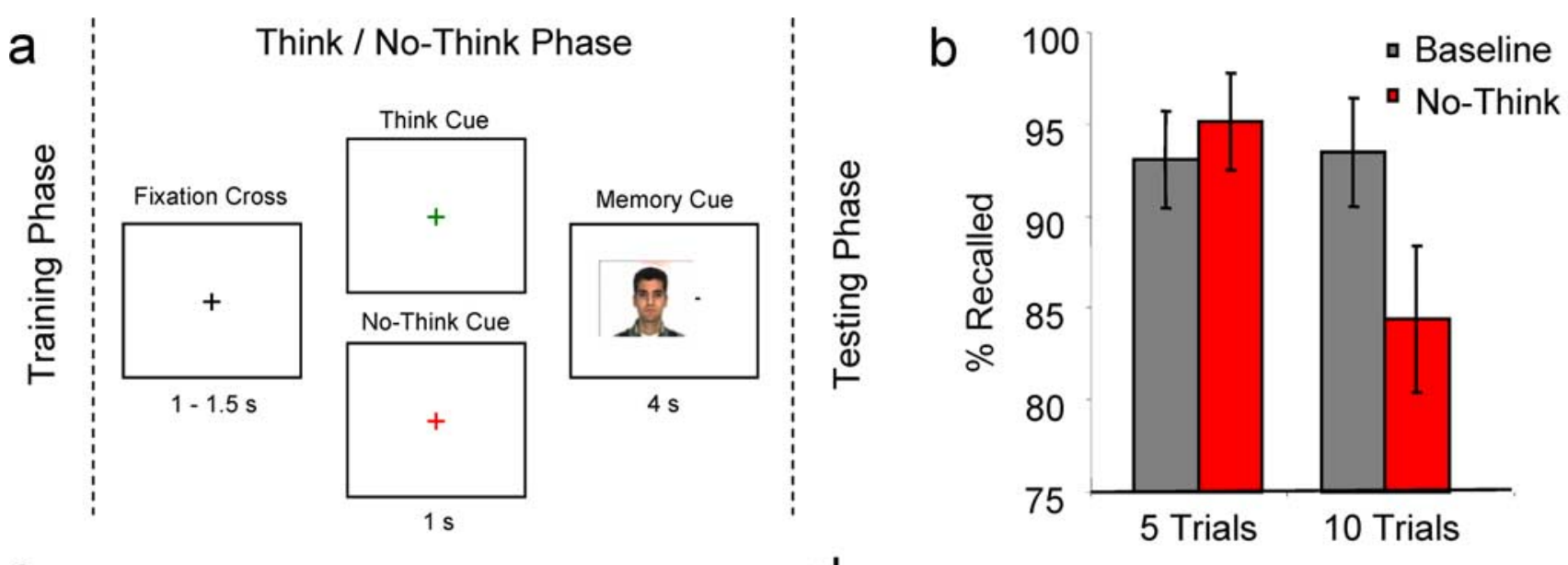

C
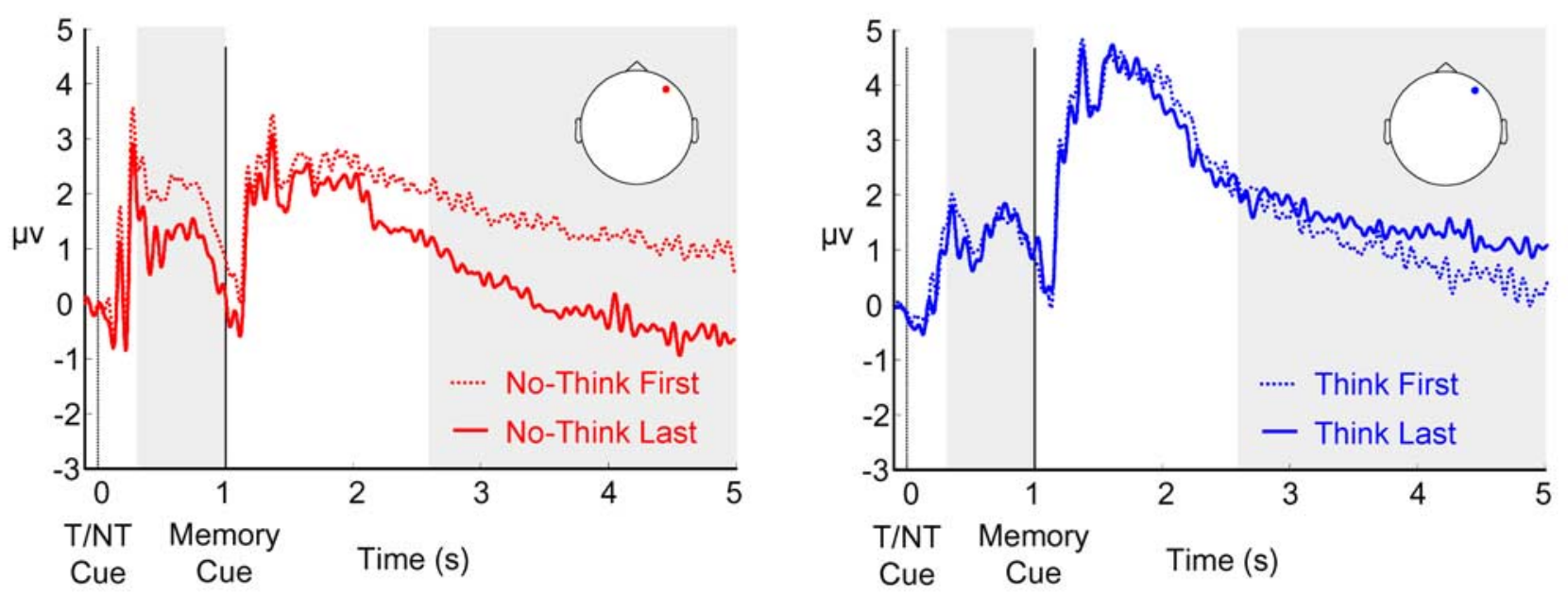

e

No-Think Think Interaction

First vs. Last First vs. Last T/NT x Rep.
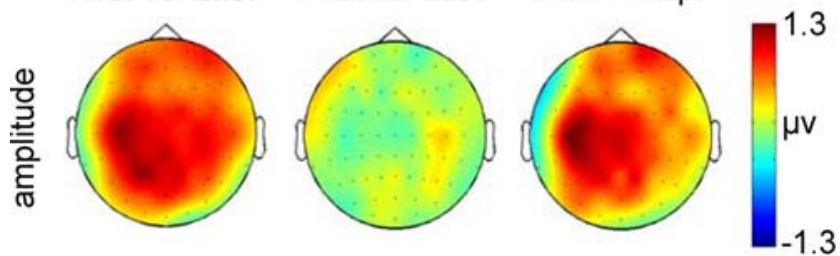

No-Think

Think First vs. Last First vs. Last
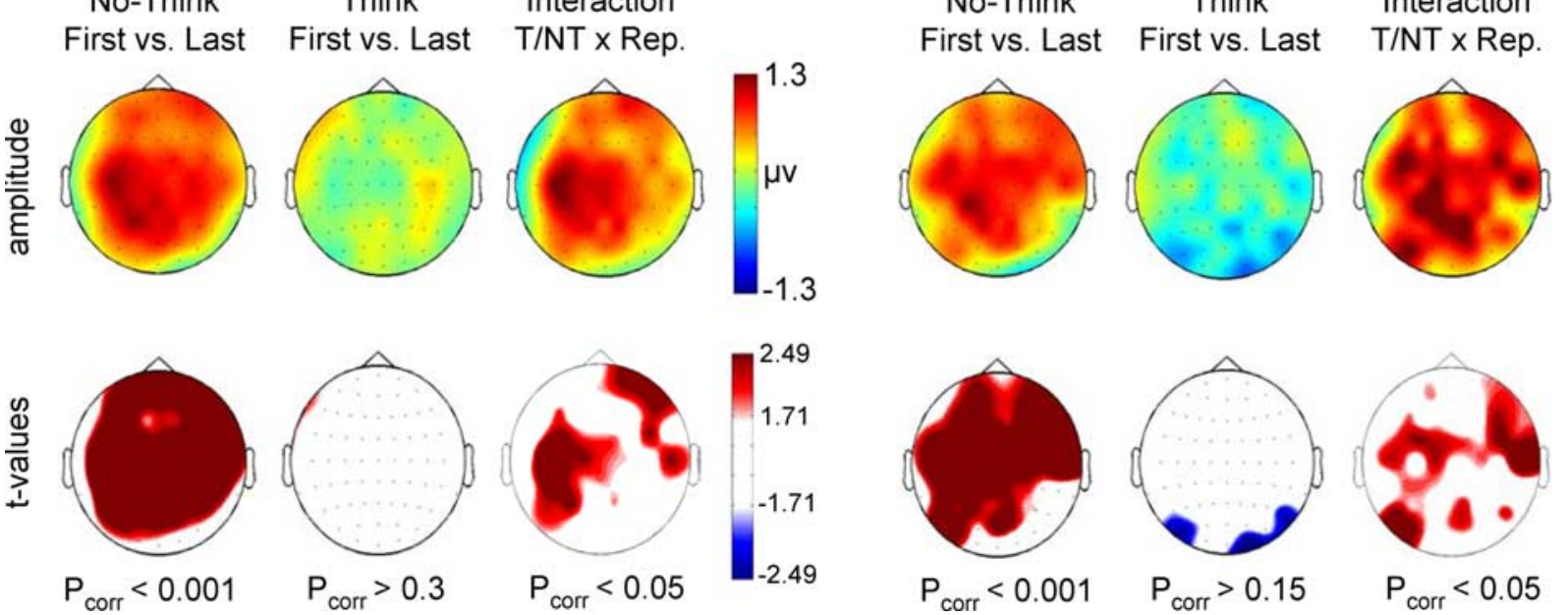
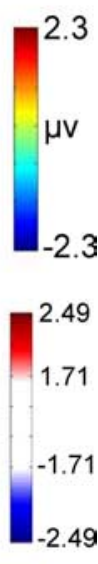

Figure 1. $\quad \boldsymbol{a}$, Experimental procedure. During the training phase participants studied several face-word pairs and engaged in a T/NT phase thereafter. In the T/NT phase, the fixation cross changed its color after a baseline period, predicting a forthcoming T trial (green) or a forthcoming NT trial (red). Next, the cue item of a previously studied face-word pair was presented, and participants were asked to either remember (T condition) or actively suppress any thought (NT condition) about the target word. The T/NT phase was followed by a cued recall test, during which all previously studied items should be recalled. $\boldsymbol{b}$, Behavioral results. In the cued recall test, forgetting (baseline - NT) was absent after five NT trial repetitions, but was present after 10 NT trial repetitions. Error bars represent SE. c, ERP waveforms for the first five (dotted line) and the last five (solid lines) NT trial repetitions for one representative electrode (for more electrodes, see supplemental Fig. $2 a$, available at www.jneurosci.org as supplemental material). Gray bars indicate the time windows during which significant differences emerged. $\boldsymbol{d}$, ERP waveforms for the first five (dotted line) and the last five (solid lines) T trial repetitions for one representative electrode (for more electrodes, see supplemental Fig. 1a, available at www.jneurosci.org as supplemental material). Gray bars indicate the time windows that were used for plotting the topography. $\boldsymbol{e}$, Topography of the anticipatory ERP effects plotted in amplitude (top row) and $t$ values (bottom row). $f$, Topography of the memory cue ERP effects plotted in amplitude (top row) and $t$ values (bottom row). Red colors indicate decreased positivity with increasing repetitions. $p_{\text {corr }}$ refers to the $p$ value obtained by randomization tests (see Materials and Methods). 
word database (Battig and Montague, 1969; Mannhaupt, 1983), and was chosen such that each of the 54 words belonged to a different semantic category.

\section{Procedure}

The experimental session consisted of two blocks, each including a complete T/NT procedure (training phase, T/NT phase, and testing phase) (Fig. 1a). A similar blocked design of the T/NT task was also used in a previous study (Depue et al., 2006). In each block, 27 face-word pairs served as item material, including three filler items used for practice. Eight of the 27 face-word pairs were repeated 10 times during the T/NT phase and served as NT items, and another eight face-word pairs were repeated 10 times and served as $\mathrm{T}$ items. The remaining eight face-word pairs did not occur during the T/NT phase and served as a behavioral baseline. The material was counterbalanced across the three item types and the two blocks. The specific phases of the T/NT procedure are described in more detail next.

Training phase. In each of the two blocks, participants first went through two study-test cycles. In each of the two cycles, participants studied 27 face-word pairs followed by a cued recall test, during which the face of a face-word pair was presented as a memory cue for the target word. Each study trial started with a fixation cross with variable duration (1.25-1.75 s), followed by a face-word pair for another $5 \mathrm{~s}$; participants were instructed to memorize the face-word pairs. Each test trial in the training phase consisted of a fixation cross with variable duration (1.25$1.75 \mathrm{~s}$ ), followed by a face for $4 \mathrm{~s}$; participants were instructed to use the face as a memory cue to recall the associated word, and to speak out the word as soon as they remembered it. Mean recall performance at the end of the second study-test cycle was $80.75 \%$ (SD: 11.25); no participant recalled $<50 \%$ of the target items.

Think/no-think phase. Before the T/NT phase, participants performed 15 NT practice trials during which each of the three filler items was repeated five times. This was done to familiarize participants with the NT instruction. During the NT trials, the participants were instructed to avoid thinking about the previously learned item by pushing the target item out of consciousness. The subjects were told to keep fixation on the memory cue, and they were strongly discouraged to generate other associations to the memory cue. Each trial in the T/NT phase started with a fixation cross, which turned either red or green after a variable delay (1-1.5 s), informing participants whether to prepare for thinking of a previously studied item (think: green) or prepare for not letting a previously studied item enter consciousness (no-think: red). This T/NT cue was presented for $1 \mathrm{~s}$ and was followed by a face, which was shown for $4 \mathrm{~s}$ and served as the memory cue. Each of the eight T and the eight NT items was repeated 10 times. The sequence of items was pseudorandomized, with the restriction that the same condition (T vs NT) did not occur more than three times in a row.

Testing phase. During the testing phase, all previously studied targets, except the three filler items, were tested by means of a cued recall test. A trial in the testing phase consisted of a fixation cross with variable duration (1.25-1.75 s), followed by a memory cue for $4 \mathrm{~s}$. The face of a previously studied cue-target pair was presented, and participants were instructed to speak out the word that was initially associated with the face. The analysis of the behavioral data was based only on those cuetarget pairs for which participants could recall the target item correctly in the second study-test cycle of the training phase (Anderson et al., 2004).

\section{EEG recording}

The EEG was recorded with a Brainamp MR+ amplifier (Megis, BrainVision) from $63 \mathrm{Ag}-\mathrm{AgCl}$ electrodes positioned according to the extended 10-10 system. EEGs were recorded against a reference electrode placed at $\mathrm{FCz}$, and were digitally rereferenced against the mean of the left and right mastoids. Vertical and horizontal eye movements were recorded by two additional channels to control for eye movements and blinks. Impedances were kept at $<10 \mathrm{k} \Omega$, and the sampling rate was set to $500 \mathrm{~Hz}$. Signals between 0.1 and $250 \mathrm{~Hz}$ were recorded, and a notch filter was set at $50 \mathrm{~Hz}$. Before analysis, the EEG data were corrected for EOG artifacts using calibration data to generate individual artifact coefficients and the PCA method (for details, see Ille et al., 2002). Remaining artifacts were excluded by careful visual inspection (artifacts that were related to muscle activity or residual eye movement artifacts due to poor EOG correction).

\section{ERP analysis}

To analyze ERP waveforms, the software package BESA (MEGIS Software BESA v5.1.8) and self-written MATLAB codes (The MathWorks) were used. The data were epoched from -500 to $5000 \mathrm{~ms}$ around onset of the T/NT cue. For ERP analysis only trials containing those items that were successfully recalled in the second study-test cycle during the training phase were used. ERP waveforms were computed separately for the first five and the last five trial repetitions for T and the NT condition. For the first five trial repetitions, an average of 58.6 trials (range: 41-75) and 59.2 trials (range: 44-75) remained for the $\mathrm{T}$ and the NT condition, respectively. For the last five repetitions, an average of 58.2 trials (range: $38-75$ ) and 59.2 trials (range: 47-76) remained for the $\mathrm{T}$ and the NT condition, respectively. Before statistical analysis, the ERP waveforms were baseline corrected with respect to a prestimulus interval of -100 to $0 \mathrm{~ms}$ and low-pass filtered at $15 \mathrm{~Hz}$ ( $48 \mathrm{db} /$ oct roll-off). No significant differences were observed in the prestimulus interval ( -0.5 to $0 \mathrm{~s})$, ruling out unspecific sustained activity $\left(p_{\text {corr }}>0.5\right)$ (supplemental Fig. 5, available at www.jneurosci.org as supplemental material).

For statistical analysis, pairwise comparisons between the first five and the last five trial repetitions within the T and the NT condition were performed by means of $t$ tests. To control for multiple testing, a two-stage randomization procedure was applied. In the first step, $t$ tests are calculated to investigate which electrodes show a significant difference between the two conditions ( $p<0.05$, two-tailed). In the second step, a randomization test using 2000 permutation runs is conducted. In this randomization procedure, the two conditions are interchanged randomly for each participant and each randomization run. This is done to induce noise in the data, and to investigate randomly generated effects. After each randomization run, $t$ tests are calculated for all electrodes, returning the number of electrodes that show a significant difference between the two conditions. After 2000 permutation runs, a distribution of the number of electrode sites that randomly show significant differences between the two conditions is generated. This distribution is used to determine the $p$ level of a given number of significant electrodes. This $p$ level $\left(p_{\text {corr }}\right)$ reflects how many times a given number of significant electrodes (e.g., 15) is exceeded in the permutation runs (values $>15$ ). In other words, the $p$ level reflects the probability that a given number of electrodes exhibiting a significant difference between two conditions is found by chance. This method is based on the randomization procedure proposed by Blair and Karniski (1993), and was already used in several other studies (Bäuml et al., 2008; Pastötter et al., 2008; Hanslmayr et al., 2009).

\section{Results}

\section{Behavioral results}

The behavioral results are plotted in Figure $1 b$. We found the expected effect of the NT instruction on memory performance in the test phase: recall in the NT condition was significantly reduced after $10 \mathrm{~T} / \mathrm{NT}$ trial repetitions (baseline: 93.4\%; NT: $\left.84.4 \% ; t_{(23)}=3.08 ; p<0.005\right)$; recall in the $\mathrm{T}$ condition $(93.8 \%)$ did not differ significantly from baseline $\left(t_{(23)}=-0.10 ; p>0.5\right)$. A control analysis indicated that the repetition of two T/NT blocks did not influence the behavioral results (see supplemental Analysis, available at www.jneurosci.org as supplemental material). To show that forgetting is absent after small numbers of trial repetitions we conducted a behavioral control experiment, in which we examined T/NT effects in a group of 24 subjects carrying out only $5 \mathrm{~T} / \mathrm{NT}$ trial repetitions (Fig. $1 b$ ). In accordance with previous work (Anderson and Green, 2001; Depue et al., 2006), no forgetting effects were found in this group of subjects (baseline: $93.1 \%$; NT: $95.1 \%$; $\left.t_{(23)}=-1.00\right)$. Consistently, a direct comparison of forgetting (baseline - NT) between the two groups showed that forgetting after 10 trial repetitions was sig- 
nificantly stronger than after 5 trial repetitions (9.1\% vs $-2.1 \%$; $\left.t_{(46)}=3.10 ; p<0.005\right)$. Concerning enhancement $(\mathrm{T}-$ baseline $)$ no significant difference between the two trial repetition conditions was obtained $\left(0.1 \%\right.$ vs $\left.6.4 \% ; t_{(46)}=1.43 ; p>0.15\right)$, showing that the amount of trial repetition had no impact on memory performance in the $\mathrm{T}$ condition. These findings suggest that, when investigating electrophysiological correlates of suppression-induced forgetting in the 10-trial-repetition condition, we can use the first five NT trial repetitions as an electrophysiological baseline for the last five NT trial repetitions.

\section{ERP results}

The ERP waveforms of the first five NT trial repetitions and the last five NT trial repetitions are plotted in Figure $1 c$ for one representative electrode (for more electrodes, see supplemental Fig. $1 a$, available at www.jneurosci.org as supplemental material). Two effects emerged: an anticipatory effect related to the onset of the NT cue $\left(0.3-1.0 \mathrm{~s} ; p_{\text {corr }}<0.001\right)$, and a later effect related to the onset of the memory cue $\left(2.6-5.0 s ; p_{\text {corr }}<0.001\right)$. To determine the time course of these effects, statistical comparisons were conducted for each single time point (supplemental Fig. $1 b$, available at www.jneurosci.org as supplemental material), showing that a stable significant difference emerged in the time interval before the memory cue at $\sim 0.3 \mathrm{~s}$, and in a later time interval at $\sim 2.6 \mathrm{~s}, 1.6 \mathrm{~s}$ after the presentation of the memory cue. In the T condition, no significant effect of trial repetition was found (Fig. $1 d$; supplemental Fig. 2, available at www.jneurosci.org as supplemental material).

Figure 1e shows the topography of the anticipatory effect. Positivity decreased with increasing trial repetitions in the NT condition (Fig. 1e, left), but not in the T condition (Fig. 1e, middle). A T/NT $\times$ repetition interaction analysis revealed that, compared with the $\mathrm{T}$ condition, positivity in the NT condition was selectively reduced over right frontal and left parietal electrode sites with increasing trial repetitions (Fig. 1e, right). The topography of the memory cue-related effects are plotted in Figure $1 f$. Similar to the anticipatory effect, memory cue-related positivity was reduced in the NT condition (Fig. $1 f$, left), but not in the T condition (Fig. $1 f$, middle). Again, a T/NT $\times$ repetition interaction analysis showed that the positivity in the NT condition was selectively reduced over right frontal and left centroparietal electrode sites (Fig. $1 f$, right). Positivity in the memory cue-related time window was generally higher in the T than in the NT condition ( $p_{\text {corr }}$ $<0.001$ ) (supplemental Fig. 3, available at www.jneurosci.org as supplemental material). Correlation analysis revealed that the anticipatory effect and the memory cue effect were positively related $(r=$ $0.56 ; p<0.005)$, indicating that the later, memory cue-driven effect was predictable from the earlier, anticipatory effect (supplemental Fig. $4 a$, available at www.jneurosci.org as supplemental material). Again, a control analysis revealed that the repetition of the two T/NT procedures did not influence the ERP results (see supplemental Analysis, available at www.jneurosci.org as supplemental material).

\section{Relation between ERP and behavior}

To investigate whether the electrophysiological effects were related to later forgetting, the subject sample was split into a highand a low-forgetting group based on the individual forgetting scores (baseline - NT). For the balanced median split, care was taken that the same item material was used in the high- and low-forgetting groups. For each of the two ERP effects, a two-way ANOVA with the between-subjects factor forget (high vs low forgetters) and the within-subjects factor repetition (first five vs last five repetitions) was calculated. As the dependent measure,
ERP amplitudes were averaged across those electrode sites that exhibited a significant T/NT $\times$ repetition interaction in the previous analysis. The ERP waveforms for high and low forgetters are shown in Figure $2 a$. In both time windows, high forgetters showed reduced positivity between the first and the last five trial repetitions ( $p_{\text {corr }}$ values $<0.001$ ), whereas low forgetters showed no such amplitude reductions (Fig. 2b). A significant forget $\times$ repetition interaction was found for the NT condition, for both the anticipatory effect $\left(F_{(1,22)}=8.676 ; p<0.01\right)$ and the later memory cue-driven effect $\left(F_{(1,22)}=8.73 ; p<0.01\right)$ (Fig. $\left.2 c\right)$. Importantly, in none of the two time windows was a significant forget $\times$ repetition interaction found for the $\mathrm{T}$ condition $(p$ values $>0.1)$. Although these nonsignificant interactions might not be surprising given the nonsignificant effects of repetition on the $\mathrm{T}$ condition, this pattern nevertheless demonstrates that the positivity reduction for high forgetters occurred selectively in the NT condition (Fig. 2c).

\section{Discussion}

We replicated previous behavioral work by showing that the repeated suppression of previously encoded target items can cause later forgetting of the suppressed information (Anderson and Green, 2001; Anderson et al., 2004) [for review, see Levy and Anderson (2008) and Bäuml (2008)]. We also replicated that suppression-induced forgetting can be present after a relatively high number of NT trials (e.g., 10 trials) but is typically absent after a relatively low number of such trials (e.g., five trials) (Anderson and Green, 2001; Depue et al., 2006), indicating that the neural mechanisms responsible for the later forgetting evolve over several NT trial repetitions (Depue et al., 2007). Going beyond the previous work, and comparing the ERP waveforms between the first and the second half of the NT trial repetitions, we identified two electrophysiological effects of NT trials, an early anticipatory effect and a later memory cue-related effect. The two effects were specific to the NT condition, ruling out unspecific processes, like repetition priming (Grill-Spector et al., 2006). In particular, the two effects predicted later forgetting, thus demonstrating the functional significance of these processes in the present paradigm.

This study is the first one to provide evidence for anticipatory effects of voluntary memory suppression. Compared with the first set of NT trials, the second set was marked by reduced positivity $\sim 300 \mathrm{~ms}$ after presentation of the NT instruction, but before presentation of the memory cue. This effect most likely reflects top-down driven suppression mechanisms, preparing the brain for episodic inhibition. Consistently, the early anticipatory ERP effect predicted the later, memory cue-driven ERP effect, likely to reflect suppression of the memory item itself (supplemental Fig. $4 a$, available at www.jneurosci.org as supplemental material). Although both ERP effects showed a similar topography, a direct comparison between the two effects revealed that the memory cue driven ERP effect was slightly stronger than the anticipatory ERP effect (supplemental Fig. $4 b$, available at www. jneurosci.org as supplemental material). This difference suggests that the T/NT cue prepares memory related brain areas for inhibition, which then gets stronger and turns into item directed inhibition when the memory cue is presented.

The memory-cue driven ERP effect that we observed in our data mimics the cued retrieval effect reported in several previous studies (e.g., Allan et al., 1996), which demonstrated that stronger sustained positivity indicates the successful retrieval of episodic memories. In the present study, we found a reduction in this cued-recall positivity with increasing NT trial repetitions, sug- 


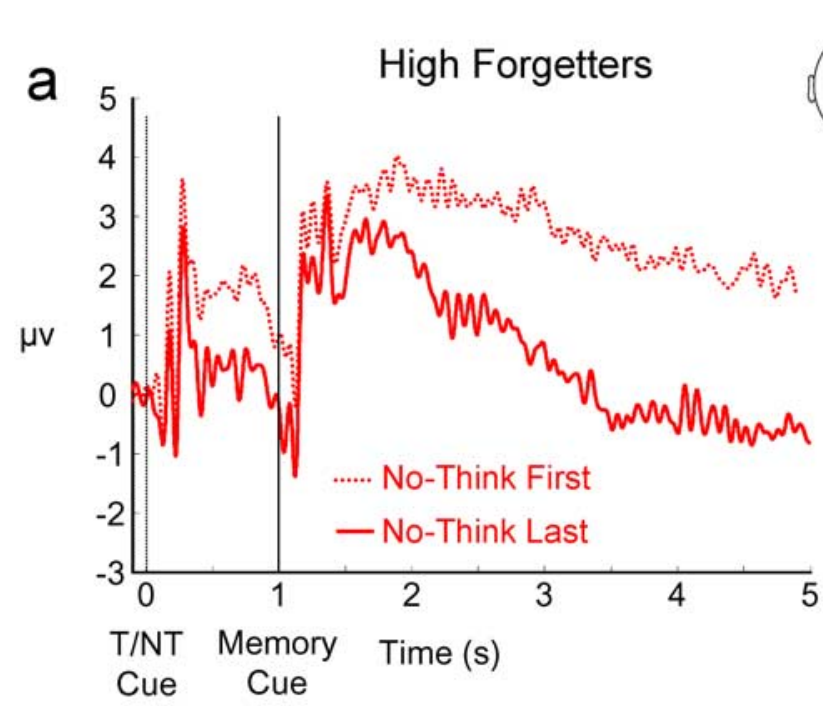

b

$$
0.3-1 \mathrm{~s}
$$

\section{$2.6-5 s$}

\section{High Forgetters Low Forgetters}

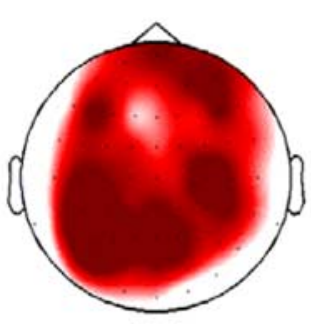

$P_{\text {corr }}<0.001$

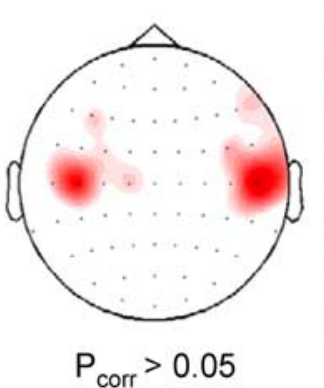

$P_{\text {corr }}>0.05$

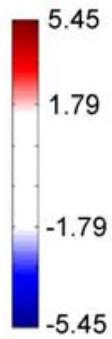

$-5.45$

C Anticipatory Effect $(0.3-1 \mathrm{~s})$

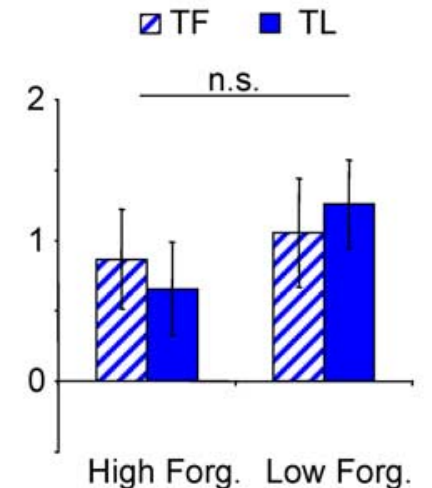

High Forg. Low Forg. $\square N T F \square N T L$

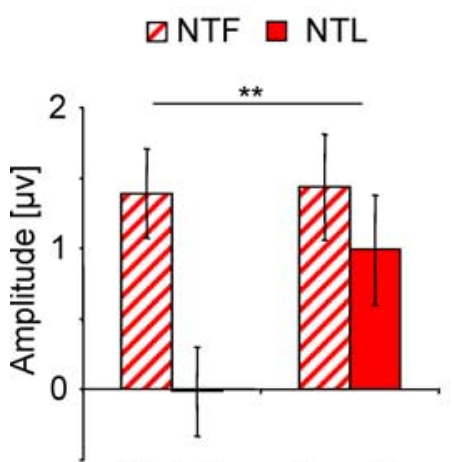

High Forg. Low Forg.

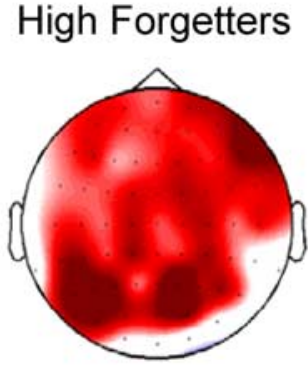

$P_{\text {corr }}<0.001$

\section{Low Forgetters}

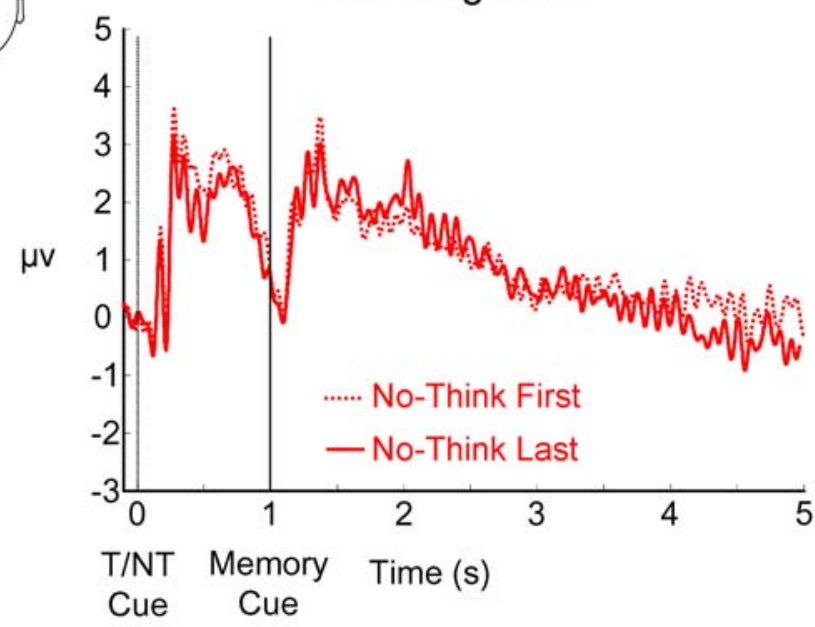

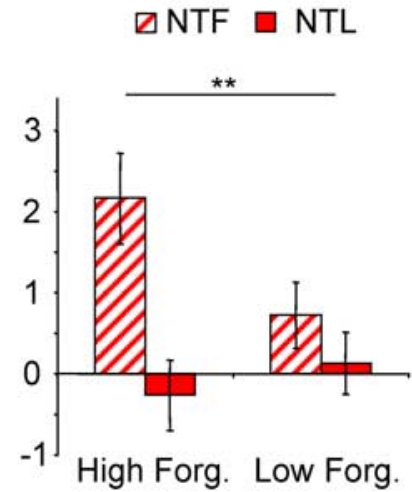

Low Forgetters

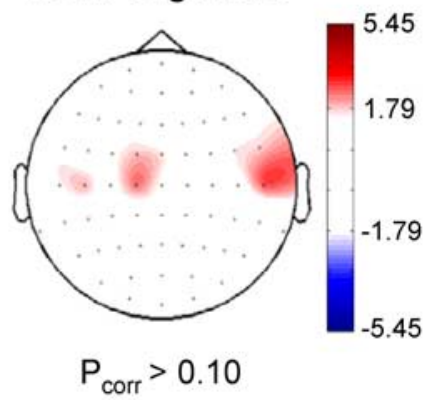

Figure 2. a, ERP waveforms for the first five (dotted line) and the last five (solid line) NT trial repetitions plotted for high and low forgetters, as deduced from a balanced median split on the forgetting scores. $\boldsymbol{b}$, Differences between the first five and the last five NT trial repetitions for high and low forgetters. The $t$ values for the early (left) and late (right) ERP effect are plotted. $p_{\text {corr }}$ refers to the $p$ value obtained by randomization tests. $c$, The mean amplitude for high and low forgetters is plotted for the no think and the think condition during the first and last five trial repetitions (NTF: no think first, NTL: no think last, TF: think first, TL: think last). High forgetters show a reduction with increasing trial repetitions in the NT condition only. Error bars represent SE. ${ }^{* *} p<0.01 ;$ n.S., nonsignificant effect.

gesting that, in NT trials, retrieval success is voluntarily downregulated, leading to memory impairment. Like the anticipatory effect, the reduced cued-recall ERP effect predicted the later forgetting. At first glance surprising, high forgetters exhibited a stronger positivity during the first five NT trial repetitions than low forgetters (Fig. $2 c$, right). This pattern, however, could reflect momentary reactivations of NT items during early NT trials (Anderson et al., 2004), which might trigger greater executive control and therefore stronger subsequent inhibition [for similar observations, see Anderson et al. (2004), Depue et al. (2007), and Kuhl et al. (2007)]. 
Both the anticipatory and the memory cue-related effect showed a right frontal and left parietal topography. Previous studies investigating effects of retrieval mode and retrieval orientation showed that sustained positivity over right frontal electrode sites is related to an episodic retrieval mode (Düzel et al., 1999), which is elicited when subjects prepare for episodic retrieval (Herron and Wilding, 2006). The results of the present study mirror these findings and demonstrate that the opposite pattern occurs when the brain prepares for episodic inhibition. The results thus point to the initiation and maintenance of an episodic inhibition mode in response to NT trial repetition.

Two recent ERP studies also addressed electrophysiological correlates during the T/NT task (Bergström et al., 2007, 2009). Contrasting T with NT trials, Bergström et al. $(2007,2009)$ found a reduced left parietal positivity to be elicited by NT items, interpreted to reflect voluntary avoidance of automatic recollection. Performing the same analysis as Bergström et al. (2007, 2009) did, we also found a reduction of the "late positive component" (LPC) (Rugg et al., 1998) beginning 500 ms after memory cue presentation $\left(p_{\text {corr }}<0.001\right)$ (supplemental Fig. 4 , available at www. jneurosci.org as supplemental material); and as in the previous work, the reduction was not related to forgetting. In contrast, it was the later positivity reduction, beginning $\sim 1.6$ s after memory cue presentation, that was related to the forgetting in the present study. Thus, while a reduction in the LPC may well reflect the avoidance of automatic recollection, the later sustained reduction of the positivity seems to reflect item suppression, and to underlie the subsequent forgetting.

Using fMRI, Depue et al. (2007) investigated how the neural correlates of voluntary suppression emerge over several T/NT trial repetitions. Their results show that activity in the right middle frontal gyrus was inversely related to activity in the hippocampus. This pattern predicted the later forgetting, and was present in the late but not the early T/NT phase. Consistently, the present results show that the physiological correlates of successful forgetting emerge with increasing trial repetitions. Although it is difficult to draw firm conclusions about neural sources from scalprecorded ERPs, the observed right frontal and left parietal distributions of the two ERP effects might reflect the activity of right prefrontal cortical areas, which downregulate the activity in memory-related processing areas (e.g., left medial temporal cortex).

To summarize, we identified two electrophysiological processes of voluntary memory suppression, an early anticipatory process and a later item-directed process. The two processes showed similar topography, were positively related, and predicted forgetting. The results suggest the existence of anticipatory mechanisms mediating voluntary memory suppression.

\section{References}

Adcock RA, Thangavel A, Whitfield-Gabrieli S, Knutson B, Gabrieli JDE (2006) Reward-motivated learning: mesolimbic activation precedes memory formation. Neuron 50:507-517.
Allan K, Doyle MC, Rugg MD (1996) An event-related potential study of word-stem cued recall. Brain Res Cogn Brain Res 4:251-262.

Anderson MC, Green C (2001) Suppressing unwanted memories by executive control. Nature 410:366-369.

Anderson MC, Ochsner KN, Kuhl B, Cooper J, Robertson E, Gabrieli SW, Glover GH, Gabrieli JDE (2004) Neural systems underlying the suppression of unwanted memories. Science 303:232-235.

Battig WF, Montague WE (1969) Category norms for verbal items in 56 categories: a replication and extension of the Connecticut category norms. J Exp Psychol Monographs 80:1-64.

Bäuml KH (2008) Inhibitory processes. In: Cognitive psychology of memory (Roediger HL, ed), pp 195-220. Oxford: Elsevier.

Bäuml KH, Hanslmayr S, Pastötter B, Klimesch W (2008) Oscillatory correlates of intentional updating in episodic memory. Neuroimage 41:596-604.

Bergström ZM, Velmans M, de Fockert J, Richardson-Klavehn A (2007) ERP evidence for successful voluntary avoidance of conscious recollection. Brain Res 1151:119-133.

Bergström ZM, de Fockert J, Richardson-Klavehn A (2009) Event-related potential evidence that automatic recollection can be voluntarily avoided. J Cogn Neurosci. Advance online publication. Retrieved February 4, 2009. doi:10.1162/jocn.2009.21075.

Blair RC, Karniski W (1993) An alternative method for significance testing of waveform difference potentials. Psychophysiology 30:518-524.

Depue BE, Banich MT, Curran T (2006) Suppression of emotional and nonemotional content in memory. Psychol Sci 17:441-447.

Depue BE, Curran T, Banich MT (2007) Prefrontal regions orchestrate suppression of emotional memories via a two-phase process. Science 317:215-219.

Düzel E, Cabeza R, Picton TW, Yonelinas AP, Scheich H, Heinze HJ, Tulving E (1999) Task-related and item-related brain processes of memory retrieval. Proc Natl Acad Sci U S A 96:1794-1799.

Grill-Spector K, Henson R, Martin A (2006) Repetition and the brain: neural models of stimulus-specific effects. Trends Cogn Sci 10:14-23.

Hanslmayr S, Spitzer B, Bäuml KH (2009) Brain oscillations dissociate between semantic and non-semantic encoding of episodic memories. Cereb Cortex. Advance online publication. Retrieved February 4, 2009. doi:10.1093/cercor/bhn197.

Herron JE, Wilding EL (2006) Neural correlates of control processes engaged before and during recovery of information from episodic memory. Neuroimage 30:634-644.

Ille N, Berg P, Scherg M (2002) Artifact correction of the ongoing EEG using spatial filters based in artifact and brain signal topographies. J Clin Neurophysiol 19:113-124.

Kuhl BA, Dudukovic NM, Kahn I, Wagner AD (2007) Decreased demands on cognitive control reveal the neural processing benefits of forgetting. Nat Neurosci 10:908-914.

Levy BJ, Anderson MC (2008) Individual differences in the suppression of unwanted memories: the executive deficit hypothesis. Acta Psychol (Amst) 127:623-635.

Martinez AM, Benavente R (1998) The AR face database. CVC Technical Report 24: June.

Mannhaupt HR (1983) Reproduktionsnormen für verbale Reaktionen zu 40 geläufigen Kategorien. Sprache und Kognition 4:264-278.

Otten LJ, Quayle AH, Akram S, Ditewig TA, Rugg MD (2006) Brain activity before an event predicts later recollection. Nat Neurosci 9:489-491.

Pastötter B, Bäuml KH, Hanslmayr S (2008) Oscillatory brain activity before and after an internal context change- evidence for a reset of encoding processes. Neuroimage 43:173-181.

Rugg MD, Mark RE, Walla P, Schloerscheidt AM, Birch CS, Allan K (1998) Dissociation of the neural correlates of implicit and explicit memory. Nature 392:595-598. 